\title{
Ignition Properties of Thermally Thin Plastics: The Effectiveness of Non- Competitive Char Formation in Reducing Flammability
}

\author{
M. I. NELSON† \\ Department of Fuel and Energy, The University of Leeds, Leeds LS2 9JT, UK \\ J. BRINDLEY \\ School of Mathematics, The University of Leeds, Leeds LS2 9JT, UK \\ A. C. MCINTOSH \\ Department of Fuel and Energy, The University of Leeds, Leeds LS2 9JT, UK
}

\begin{abstract}
The retardancy effect of char formation upon the flammability of thermally thin products is investigated. The char is formed in a single-step non-competitive scheme and is assumed to be thermally stable. The criterion for ignition is that of a critical mass flux of volatiles from the solid into the gas phase. Both steady-state and transient formulations of the model are considered. In the high activation energy limit the critical heat flux efficiency in the steady-state model is proportional to $c /(1-c)$, where $c$ is the fraction of char formed. In the transient model the efficiency in reducing the maximum heat release rate, average heat release rate, and total heat released is given by $c$ and is independent of activation energy and heat flux. The specific application that we have in mind for our model is piloted ignition in the cone calorimeter.
\end{abstract}

Keywords: cone calorimeter, critical heat flux, heat release rate, mass loss rate

\section{Introduction}

The use of synthetic polymers in buildings or construction applications is steadily increasing and every year more emphasis is placed on the hazards that result from the burning of such materials. The majority of polymercontaining end products must now pass regulatory tests and there is therefore considerable interest in the design of materials that can pass such tests. Although halogenated flame retardant systems have proven very effective, environmental concerns have prompted the development of alternative flame retardant systems. Research into new fire-retardant systems

$\dagger$ Requests for reprints should be sent to M. I. Nelson, School of Mathematics and Statistics, University of New South Wales, Canberra ACT 2600, AUSTRALIA. 
covers a broad range of approaches, including the systematic investigation into combinations of additives that promote synergy[25], intumescent systems[4], [10], [25], char formation[10], [25], [27], the use of heat sink additives such as aluminium trihydrate and magnesium hydroxide etc. Kashiwagi has identified char formation as the most promising of these and has reviewed the benefits of char formation in improving the fire resistance of polymers[13].

The advantages of char formation are:

- $\quad$ Reduced mass of volatiles.

Part of the carbon (and hydrogen) stays in the condensed phase, reducing the mass of volatile combustible degradation fragments evolved.

- Thermal insulation.

As the polymer degrades a char layer is formed. The low thermal conductivity of this layer enables it to provide thermal insulation, absorbing some of the heat input and therefore reducing the heat flux reaching the virgin polymer[5], [11]. In addition, re-radiation losses are significantly increased, further protecting the polymer. Both of these processes reduce thermal degradation.

- Obstruction of combustible gases.

A charred surface may act as a physical barrier, obstructing the flow of combustible gases generated from the degradation of the underlying unburnt material, and hindering the access of oxygen to the surface of the polymer[5].

We use the critical massflux approach to investigate the effectiveness of char-formation in reducing flammability. A simple model is used in which ignition or extinction of a flame occurs when the mass flux of volatiles from the solid passes through a critical value. This method has been used to investigate the flammability of thermoplastics and to model inert and heat-sink retardants[18], [19], [20], [21].

The charring of a thermally-thick sample leads to the formation of three zones (figure 1 (b)): a charred zone, containing no polymer; a pyrolysis zone, containing both char and polymer; and a polymer zone, comprising only virgin polymer. The physical and chemical behaviour of these zones can be quite complicated and distinct from one another. For example, the char layer may be anisotropic, and properties such as porosity and thermal conductivity can be significantly different in each layer. The burning of synthetic polymers introduces another complication: the introduction of a moving boundary. Unlike wood, the sample size is not constant through the burn. In practice the pyrolysis layer may be very thin, separating two 
zones, comprising respectively polymer and char only. If the sample is thermally-thin, then it effectively comprises the pyrolysis zone.

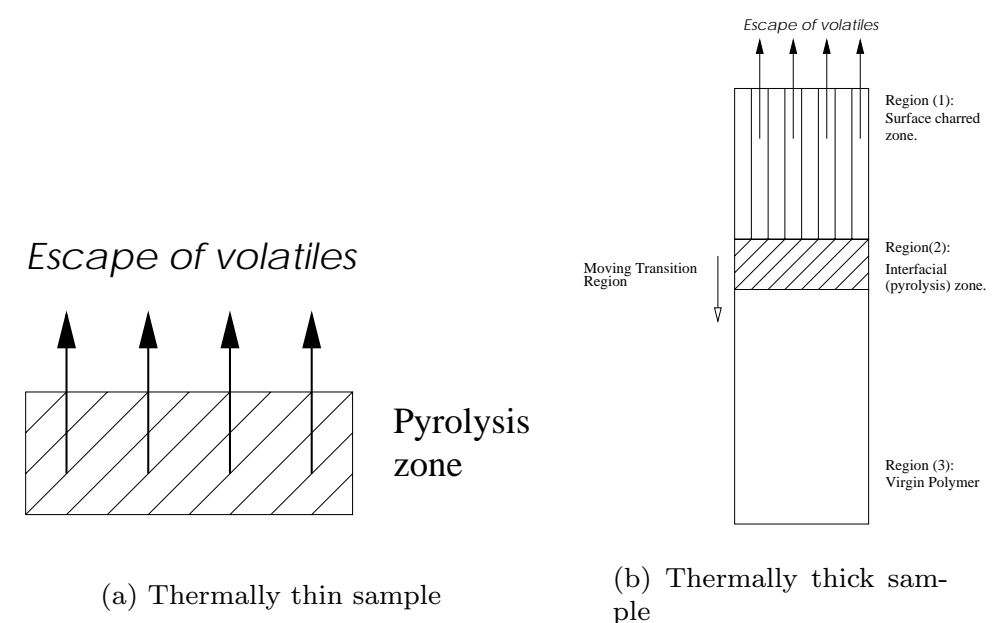

ple

Figure 1. The physical structure of a charring material: (a) one-zone structure in a thermally thin sample; (b) three zone structure in a thermally thick sample.

We consider the limiting case of thermally thin behaviour. Thus only the pyrolysis zone is present and there is neither a temperature gradient through the sample nor does the char act as a barrier. Although the assumption of thermally thin behaviour considerably simplifies the model there are end products to which it is a good approximation, e.g. clothing, curtains, drapiers, electronic circuit boards, paper etc. Thus thermally-thin behaviour is worthy of study in its own right.

\subsection{The chemistry of char formation}

We distinguish between two general mechanisms, competitive and noncompetitive, of char-formation. By non-competitive char formation we mean the scheme

$$
\mathcal{M}_{1} \rightarrow c \cdot \mathcal{C}_{1}+(1-c) \mathcal{V}
$$


where $\mathcal{M}_{1}$ is the polymer, $\mathcal{C}_{1}$ is char and $\mathcal{V}$ represents gaseous volatiles. By competitive char formation we mean the reaction scheme

$$
\begin{aligned}
& \mathcal{M}_{1} \rightarrow \mathcal{V}, \\
& \mathcal{M}_{1} \rightarrow c \cdot \mathcal{C}_{1}+(1-c) \mathcal{V} .
\end{aligned}
$$

In the non-competitive scheme the fraction of char formed is independent of the sample's heating history and is $c(0 \leq c \leq 1)$. In the competitive scheme it depends upon the heating history and is at most $c$. The attraction of non-competitive char formation is that only three parameters are required to define the reaction kinetics, whereas competitive char formation requires five. It is much more difficult to investigate parameter space in a meaningful way for the competitive scheme.

Although highly simplified, the competitive mechanism represents a prototype scheme in which char formation is based upon competition between dehydration to char, equation (3), and depolymerisation, equation (2). The detailed chemistry of char formation is more complicated than either of these schemes. However, detailed chemistry can only be incorporated into models when reliable kinetic parameters are available for all the reactions in a mechanism. This is almost invariably not the case. The few kinetic models for which parameter values are available are almost exclusively for cellulosic materials. Kinetic models for the pyrolysis of cellulose in nonoxidising environments have been reviewed by Di Blasi[3]. Kandola et al[12] discuss in detail the chemistry of cellulose pyrolysis, emphasising the implications for the design of effective fire retardants.

As a further simplification we assume that the char is thermally stable. This is the ideal situation as a flammable material is converted to a nonflammable material.

\subsection{Mathematical models of charring}

The development of one-dimensional (1-D) models for wood pyrolysis dates back to Bamford et al[2]. Starting with Kung[14], detailed models have been developed describing the charring of wood. Di Blasi[3] has reviewed subsequent development in this field. More recent models include those of Ritchie et al[22] and Yuen et al[28], the latter contains a succinct review of the development of 1-D, 2-D and 3-D models. These models often employ temperature dependent thermal properties and modelling assumptions specific to wood. For instance, anisotropic properties due to the grain structure of wood influence heat and mass transfer during pyrolysis. The existence of a consolidated porous structure means that the transport of 
gases and vapour can be modelled using Darcy's Law. Such detailed models have little application to the charring of non-cellulosic materials.

There have been few investigations of charring in non-cellulosic materials. In particular, there has been very little work investigating the efficiency of char formation in reducing flammability. Note that in the models described in sections 1.2.1 \& 1.2.2 the pyrolysis gases reach the exposed surface as soon as they are formed and that once the char is assumed to be thermally stable.

\subsubsection{Thermal pyrolysis models (infinite rate kinetics)}

In the thermal pyrolysis approach char-formation occurs at a fixed temperature and at a moving boundary separating regions of char and virgin polymer.

Chen et al [6] consider the pyrolysis of a one-dimensional charring slab. The model is reduced from two partial differential equations, one in each of the char and virgin polymer layers, to four ordinary differential equations, two in each layer, by assuming exponential temperature profiles within the layers. The heat-loss mechanism on the heated surface is purely radiative. This model was subsequently used to calculate material pyrolysis properties from flammability measurements under an inert atmosphere[7]. The latter paper shows that the thermal capacity of the char $\left(\rho_{c} c_{p_{c}}\right)$ has a negligible effect on the mass loss rate. This is explained by identifying radiative heat losses from the hot char as the dominant mechanism in reducing he pyrolysis rate. In these papers the sample is assumed to be of fixed length throughout the burn.

Leung et al[15] model a char-forming ablation process in a semi-infinite solid under an inert atmosphere. Three heat-loss mechanisms on the heated boundary are considered: no heat-loss, purely convective, and purely radiative. For all three mechanisms the mass-loss rate increases to a global maximum before decreasing to zero. This happens because, as the amount of char builds up, a greater proportion of the heat input is used in raising the heat content of the char layer. The density of the char has little effect on the mass-loss rate, and no effect in the limiting case of no heat-loss. The maximum mass-loss rate increases with increasing thermal conductivity of the char. A subsequent paper[16] investigates how the non-combustible substrate base effects the mass loss rate. Pyrolysis of char-forming and non char-forming polymers under an inert atmosphere is studied under the assumption that there is no heat loss on the heated boundary. The sample length is not fixed during the burn. Thus in the char-forming case there are two moving boundaries: the heated boundary and the char-polymer 
boundary. For thermally-thin samples the mass-loss rate increases monotonically until the polymer is completely pyrolysed. For thermally thick samples the mass-loss rate increases to a local maximum before decreasing to a local minimum. Eventually the polymer layer becomes thermally thin and at this point the mass-loss rate increases to a global maximum at the end of ablation. The global maximum decreased with increasing sample size. Although the substrate-pyrolysis model[16] could be used to investigate the effect of char-formation in reducing mass-loss rate, the exclusion of heat-loss mechanisms on the heated boundary is a serious weakness.

Staggs [24] considers a char-forming ablation process in which there are no heat losses on the heated boundary and the temperature is constant on the rear boundary. The model is reduced from two partial differential equations, one in each layer, to two ordinary differential equations, one for the mass-loss rate and one for the thermal penetration depth, by assuming polynomial temperature distributions.

\subsubsection{Finite rate kinetics}

Historically much of the work on charring materials has been concerned with wood. However, in the 1960s there was considerable interest in the potential use of ablating materials to protect a space vehicle against overheating when re-entering the earth's atmosphere. An early model is due to Matsumoto et al[17] who modelled the decomposition of charring materials subject to very large heat flows, including the heterogeneous oxidation of the char layer. The ablating material is divided into three zones (char layer, pyrolysis zone and virgin polymer) and three sets of partial differential equations are used to describe the regions. A defect in this model is the absence of heat-loss mechanisms on the char surface.

The pioneering work of Kung[14] investigates the dependence of the massloss rate upon the thermal conductivity of the char using a non-competitive scheme. There is a pronounced increase in the mass-loss rate with increasing thermal conductivity, this is particularly noticeable in the global maximum. For thermally-thick samples the mass loss rate initially increases to a local maximum before decreasing to a local minimum. Eventually the polymer layer becomes thermally thin and the mass loss rate increases to a global maximum before finally decreasing towards zero. For thermally thin samples the mass loss rate curve exhibits one global maximum. This type of behaviour is also seen in a thermal pyrolysis model[16]. However, there is a subtle difference: in the thermal pyrolysis models the global maximum occurs at the end of the burn, in the finite-rate kinetics model the mass-loss rate decays to zero after the global maximum. 
Kung suggests that in thermally thick samples the decline in mass loss rate after the first local maximum is primarily due to convective heat losses within the material caused by the outward flow of the volatiles. In fact this decrease is a structural effect entirely due to the char layer[16], which acts as an insulating layer for heat conduction and inhibits mass transfer of decomposition products.

Sibulkin[23] investigates how the heat of gasification varies during the burning of charring materials using a non-competitive scheme. The mass loss rate increases to a local maximum, decreases to a local minimum before increasing towards the end of the burn as the sample becomes thermally thin. Sibulkin calculates that the net flux into the solid decreases by $20 \%$ of its initial value after the char layer is formed because of increased surface heat losses - the surface temperature of a charring material is considerable higher than that of an equivalent non-charring material.

Staggs[24] considers competitive char formation in thermally thick samples under an inert atmosphere. The mass-loss rate is found to be sensitive to the rate of char formation and is greatly reduced by ensuring that the char-forming reaction switches on before the volatile-forming step. Chars with low density and thermal conductivity are the most beneficial at providing thermal protection.

\subsubsection{Comments on literature review}

The evolution of the mass-loss rate with time is understood for both thermally thin and thermally thick materials. However, there has been no attempt to systematically quantify the contributions made by specific physical mechanisms to the decreased flammability of char-forming materials. Moreover there is some inconsistency in the results. Chen et al[7] state that the thermal capacity of the char $\left(\rho_{c} c_{p_{c}}\right)$ has a negligible effect on mass-loss rate. Leung et al[15] also notes that char density has little effect on mass loss rate. However, Staggs[24] identifies low density as a desirable property in providing enhanced thermal protection. Other authors consider the pyrolysis of a given material and do not investigate the dependence of flammability upon physical properties, as these are assumed to be fixed. 


\section{Description of the Model}

\subsection{Physics and chemistry of the model}

The test sample is modelled as a thermally-thin slab. By thermally-thin we mean that heat absorbed on the top surface of the solid slab penetrates its thickness sufficiently rapidly that there is no significant temperature gradient through the material depth. Thus there is a uniform temperature profile and spatially uniform mass-fraction of char and polymer. The assumption of thermally-thin behaviour means that our model does not apply to strongly intumescent char-forming processes in which there is a significant increase in volume.

The rear surface and the sides of the sample are assumed to be perfectly insulated. Heat transfer processes are therefore restricted to the upper surface of the sample, which is exposed to a uniform heat flux. Heat is exchanged at this surface by convection and radiation. These assumptions are appropriate in many practical situations, notably cone calorimeter testing.

The decomposition of the polymer is modelled by a non-competitive scheme. The char is assumed to be thermally stable. Degradation products are assumed to be transported instantaneously to the sample surface.

The flame is not directly modelled, except that heat-transfer between the flame and the upper surface of the test material is assumed to be governed by the mass flux of volatiles from the test material, equation (11). When this mass flux is below criticality the temperature of the volatiles is assumed to be at ambient. When the mass flux of volatiles is greater than criticality the flame is 'turned on'.

\subsection{Formulation of the pyrolysis kinetics}

In investigating polymer combustion it has proven advantageous to formulate the reaction rate using the concept of 'characteristic temperature'[19]. In this

$$
k=\frac{\mathcal{H} E_{s}}{R T_{c}^{2}} \exp \left[\frac{E_{s}}{R}\left(\frac{1}{T_{c}}-\frac{1}{T}\right)\right],
$$

where $T_{c}$ is the characteristic temperature measured in a thermogravimetric experiment [9] and $\mathcal{H}$ is an experimental parameter. Note that for a fixed characteristic temperature, increasing the activation energy narrows the temperature range over which pyrolysis occurs. In the limit $E_{s} \rightarrow \infty$ a thermal pyrolysis model is obtained. 


\subsection{Characterizing the flammability of a product}

There are many ways in which the flammability of a product can be assessed. These include the critical heat flux, the rate of flame spread, the time-to-ignition, the heat release rate, the toxicity of the combustion gases and the amount of smoke generated. In this paper we characterise flammability by the critical heat flux $\left(\mathcal{L}_{c r}\right)$, the peak heat release rate, the average heat release rate during the burn, and the total heat released during the burn.

The critical heat flux is the smallest external heat flux which will ignite a material. If the maximum heat flux in a fire scenario is known, it can be used as a means of selecting permissible materials. It has also been used to calculate permissible building separation distances[8]. Heat release rate has been identified as being the single most important variable in characterizing product flammability and their fire hazard[1]. For thermally thin samples the heat release rate is characterised by its peak value.

It is usually assumed, in both modelling and the analysis of experimental data, that gas-phase kinetics occur on a faster timescale than the degradation kinetics. The rate of heat release is therefore controlled by the flow of volatiles into the flame. Since we assume that the char is thermally stable the heat release rate is essentially the same as the mass-loss rate (see equation (18) \& (19)). Therefore the maximum mass-loss rate corresponds to the peak heat release rate.

In order to investigate the efficiency of char-formation in reducing flammability it is necessary to chose a reference datum. The natural reference for a fire-retarded material is the non fire-retarded material. For this theoretical study our reference is a non charring material $(c=0)$ with the same physical and chemical properties as the char forming material $(c \neq 0)$. For a fixed value of the char fraction we refer to the equivalent non-charring material as the 'comparison material'.

We define the (percentage) efficiency $(\mathcal{E})$ of char formation for for a given flammability parameter $\mathcal{P}$ to be

$$
\mathcal{E}_{\mathcal{P}}=100\left(\frac{\mathcal{P}(c)}{\mathcal{P}(c=0)}-1\right),
$$

where $\mathcal{P}(c)$ is the value of the flammability parameter when the char fraction formed is $c$ and $\mathcal{P}(c=0)$ is the value of the flammability parameter when the char fraction formed is 0 . We define the fractional efficiency as $\mathcal{E}_{\mathcal{P}} / 100$. 


\section{Model Equations}

\subsection{Dimensional model}

The system we study is

$$
\begin{aligned}
& \text { Polymer degradation } \\
\frac{\mathrm{d} \mathcal{M}_{1}}{\mathrm{~d} t}= & -\frac{\mathcal{H} E_{s}}{R T_{c}^{2}} \exp \left[\frac{E_{s}}{R}\left(\frac{1}{T_{c}}-\frac{1}{T}\right)\right] \mathcal{M}_{1} \\
& \text { Char formation } \\
\frac{\mathrm{d} \mathcal{C}_{1}}{\mathrm{~d} t}= & c \cdot \frac{\mathcal{H} E_{s}}{R T_{c}^{2}} \exp \left[\frac{E_{s}}{R}\left(\frac{1}{T_{c}}-\frac{1}{T}\right)\right] \mathcal{M}_{1} \\
& \text { Energy equation for the solid } \\
\left(c_{p_{s}} \mathcal{M}_{1}+c_{p_{c}} \mathcal{C}_{1}\right) \frac{\mathrm{d} T}{\mathrm{~d} t}= & Q_{s} \frac{\mathcal{H} E_{s}}{R T_{c}^{2}} \exp \left[\frac{E_{s}}{R}\left(\frac{1}{T_{c}}-\frac{1}{T}\right)\right] \mathcal{M}_{1}+ \\
& \alpha_{s}\left(1-\alpha_{f}\right) S \mathcal{L}-S \chi\left(T-T_{f}\right)+ \\
& \sigma\left(\left[1-\mathcal{F}_{f . s}\right] \alpha_{s} T_{a}^{4}-\epsilon_{s} T^{4}\right) S+\alpha_{s} \epsilon_{f} \mathcal{F}_{f . s} \sigma T_{f}^{4} S,
\end{aligned}
$$

Mass flow from the solid phase into the gas phase

$$
\dot{m}=(1-c) \frac{\mathcal{H} E_{s}}{R T_{c}^{2}} \exp \left[\frac{E_{s}}{R}\left(\frac{1}{T_{c}}-\frac{1}{T}\right)\right] \mathcal{M}_{1},
$$

Rate of heat release

$$
\dot{\Delta}=Q_{c, e} \dot{m}
$$

Flame temperature model

$$
T_{f}= \begin{cases}T F & \text { if } \dot{m} \geq S \dot{m}_{c r}^{\prime \prime} \\ T_{a} & \text { if } \dot{m}<S \dot{m}_{c r}^{\prime \prime},\end{cases}
$$

Initial conditions

$$
\mathcal{M}_{1}(t=0)=\rho_{s} V_{0}, \quad \mathcal{C}_{1}(t=0)=0, \quad T(t=0)=T_{a} .
$$

Observe that the density of the char does not appear in these equations. Therefore for thermally thin materials it has no effect on the flammability of the system.

Equations (6) \& (7) model the degradation of the polymer and the formation of char respectively.

Equation (8) is the energy equation. The term in the parentheses on the left hand side of the equation is the total heat capacity of the solid. This changes as polymer is converted to char and volatiles. The first term on the right-hand side is the rate of gasification. This is the product of the pyrolysis rate $(k)$, and the heat of gasification. The second term is the heat 
input from the external irradiance source, allowing for the absorption of some energy by the layer of volatiles above the surface of the sample. The third term models the convective and conductive heat exchange between the sample and the volatiles. The fourth and fifth terms give the net radiative exchange between the sample and its surroundings.

Equations (9) \& (10) give the mass flux of volatiles from the solid into the gas phase and the rate of heat release rate respectively. Since the char is assumed to be thermally stable these expressions depend only upon the rate of polymer pyrolysis. Consequently the latter is a constant multiplier of the former. Thus we freely interchange between the phrases 'maximum mass loss rate' and 'peak heat release rate'.

Equation (11) is the flame sub-model. Note that the flame-is turned on (off) as the mass flux increases (decreases) through a critical value.

Equation 12) provides the initial conditions for the mass of polymer and char and the initial temperature of the system.

Equations (6-12) define the transient formulation for non competitive char formation in thermally thin samples. The peak heat release rate is the maximum value obtained by equation (10) during the burn. The total heat release during the burn is given by

$$
\mathcal{H} \mathcal{R} \mathcal{R}_{T}=Q_{c, e}\left(\mathcal{M}_{1, \text { ign }}-\mathcal{M}_{1, \text { ext }}\right),
$$

where $\mathcal{M}_{1, \text { ign }}$ is the mass of the polymer at ignition and $\mathcal{M}_{1, \text { ext }}$ is the mass at extinction. The average heat release rate is the quotient of the total heat released and the burn time

$$
\mathcal{H R}_{\mathcal{R}}=\frac{Q_{c, e}\left(\mathcal{M}_{1, i g n}-\mathcal{M}_{1, \text { ext }}\right)}{t_{\text {ext }}-t_{\text {ign }}},
$$

where $t_{i g n}$ is the ignition time and $t_{e x t}$ is the extinction time.

\subsection{Non-dimensionalized model}

Using the non-dimensionalized variables $\mathcal{M}_{1}^{*}$, the reduced fuel mass, $\mathcal{C}_{1}^{*}$, the reduced char mass, $T^{*}$, the reduced temperature, $\dot{m}^{*}$, the non-dimensionalized mass flux rate, $\dot{\Delta}^{*}$, the non-dimensionalized rate of heat release, $T_{f}^{*}$, the reduced flame temperature, and $t^{*}$, the non-dimensionalized time-scale, 
equations (6-12) are written as

Non-dimensionalized polymer degradation

$$
\frac{\mathrm{d} \mathcal{M}_{1}^{*}}{\mathrm{~d} t^{*}}=-\frac{Y_{s}^{*} E_{s}^{*}}{T_{c}^{*^{2}}} \exp \left[E_{s}^{*}\left(\frac{1}{T_{c}^{*}}-\frac{1}{T^{*}}\right)\right] \mathcal{M}_{1}^{*},
$$

Non-dimensionalized char formation

$$
\frac{\mathrm{d} \mathcal{C}_{1}^{*}}{\mathrm{~d} t^{*}}=c \cdot \frac{Y_{s}^{*} E_{s}^{*}}{T_{c}^{*^{2}}} \exp \left[E_{s}^{*}\left(\frac{1}{T_{c}^{*}}-\frac{1}{T^{*}}\right)\right] \mathcal{M}_{1}^{*},
$$

Non-dimensionalized energy equation for the solid

$$
\begin{aligned}
\left(\mathcal{M}_{1}^{*}+c_{p}^{*} \mathcal{C}_{1}^{*}\right) \frac{\mathrm{d} T^{*}}{\mathrm{~d} t^{*}}= & Q_{s}^{*} \frac{Y_{s}^{*} E_{s}^{*}}{T_{c}^{*^{2}}} \exp \left[E_{s}^{*}\left(\frac{1}{T_{c}^{*}}-\frac{1}{T^{*}}\right)\right] \mathcal{M}_{1}^{*}+ \\
& \alpha_{s}\left(1-\alpha_{f}\right) \mathcal{L}^{*}-\left(T^{*}-T_{f}^{*}\right) \\
& +\alpha_{s}\left[1-\mathcal{F}_{f . s}\right] \sigma^{*}-\epsilon_{s} \sigma^{*} T^{*^{4}}+\alpha_{s} \epsilon_{f} \mathcal{F}_{f . s} \sigma^{*} T_{f}^{*^{4}},
\end{aligned}
$$

Non-dimensionalized mass flux from the solid phase into the gas phase

$$
\dot{m}^{*}=\frac{1-c}{m_{t}^{*}} \cdot \frac{Y_{s}^{*} E_{s}^{*}}{T_{c}^{*^{2}}} \exp \left[E_{s}^{*}\left(\frac{1}{T_{c}^{*}}-\frac{1}{T^{*}}\right)\right] \mathcal{M}_{1}^{*},
$$

Non-dimensionalized rate of heat release

$$
\dot{\Delta}^{*}=\dot{m}^{*},
$$

Non-dimensionalized flame temperature model

$$
T_{f}^{*}= \begin{cases}T F^{*} & \text { if } \dot{m}^{*} \geq 1 \\ 1 & \text { if } \dot{m}^{*}<1,\end{cases}
$$

Initial conditions

$$
\mathcal{M}_{1}^{*}\left(t^{*}=0\right)=1 \quad \mathcal{C}_{1}^{*}\left(t^{*}=0\right)=0, \quad T^{*}\left(t^{*}=0\right)=1
$$

\subsection{Non-dimensionalized steady-state model}

The steady-state model is obtained from the transient model by ignoring degradation of the polymer. Thus $\mathcal{M}_{1}^{*}\left(t^{*}\right)=1$ and $\mathcal{C}_{1}^{*}\left(t^{*}\right)=0$. The temperature of the system evolves to a steady-state which is obtained by setting the temperature derivative equal to zero. The model therefore reduces to the algebraic equation

$$
\begin{aligned}
0= & Q_{s}^{*} \frac{Y_{s}^{*} E_{s}^{*}}{T_{c}^{*^{2}}} \exp \left[E_{s}^{*}\left(\frac{1}{T_{c}^{*}}-\frac{1}{T^{*}}\right)\right]+\alpha_{s}\left(1-\alpha_{f}\right) \mathcal{L}^{*}-\left(T^{*}-T_{f}^{*}\right) \\
& +\alpha_{s}\left[1-\mathcal{F}_{f . s}\right] \sigma^{*}-\epsilon_{s} \sigma^{*} T^{*^{4}}+\alpha_{s} \epsilon_{f} \mathcal{F}_{f . s} \sigma^{*} T_{f}^{*^{4}},
\end{aligned}
$$

in conjunction with equations (18-20).

The steady-state assumption has a negligible effect on the critical heat flux calculation; prior to ignition the rate of decomposition is very small, 
consequently the mass of material remaining at ignition in the transient model is very close to its initial (steady-state) mass[19], [20]. The peak heat release rate is approximately $20 \%$ higher in steady-state models compared to the equivalent transient model, but the general trends are the same. The results of sections $4 \& 5$ show that the assumption of steady-state behaviour has very little effect on the efficiency of char formation in reducing the peak heat release rate.

\section{Steady-State Results}

In sections $4.1 \& 4.2$ we investigate the effect that char formation has upon the critical heat flux and the maximum mass loss rate (peak heat release rate) respectively. Note that the critical heat flux is the value of $\mathcal{L}^{*}$ at which the non-dimensionalized mass-loss rate, equation (18), just reaches a value of one. In figures 2-6 we use dimensional instead of non-dimensional values as these are easier to interpret.

\subsection{Critical heat flux}

\subsubsection{Numerical results}

Figure 2 (a) shows how the critical heat flux depends upon the degradation kinetics for non-charring materials. Over the parameter range shown the critical heat flux decreases with increasing activation energy, though for curve (2) there is little variation. We define an average critical heat flux which is a function of the characteristic temperature only by

$$
\overline{\mathcal{L}_{c r}}\left(T_{c}\right)=\frac{10^{-3}}{160} \int_{E_{s}=80 \times 10^{3}}^{E_{s}=240 \times 10^{3}} \mathcal{L}_{c r}\left(E_{s}, T_{c}\right) \mathrm{d} E_{s} .
$$

Figure 2 (b) shows the variation of average critical heat flux and the corresponding standard deviations. Although the standard deviation increases with critical heat flux it is always smaller than the experimental uncertainty in calculating the critical heat for any one of the materials associated with the average[26]. Therefore in what follows we do not indicate the standard deviation.

Figure 3 shows how the critical heat flux and the critical heat flux efficiency vary with activation energy and the char fraction. It is only when the char fraction is 0.6 that the increase in critical heat flux becomes marginally significant from a practical perspective, and then only for low activation 


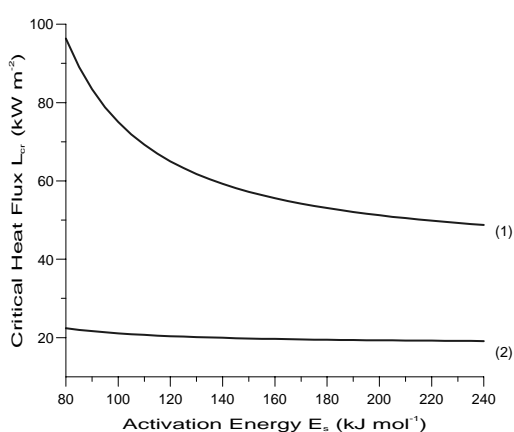

(a) Variation of critical heat flux $\left(\mathcal{L}_{c r}\right)$ with activation energy $\left(E_{s}\right)$ : (1) $T_{c}=780 \mathrm{~K}, \rho=500 \mathrm{~kg} \mathrm{~m}^{-3} ;(2)$ $T_{c}=580 \mathrm{~K}, \rho=2000 \mathrm{~kg} \mathrm{~m}^{-3}$.

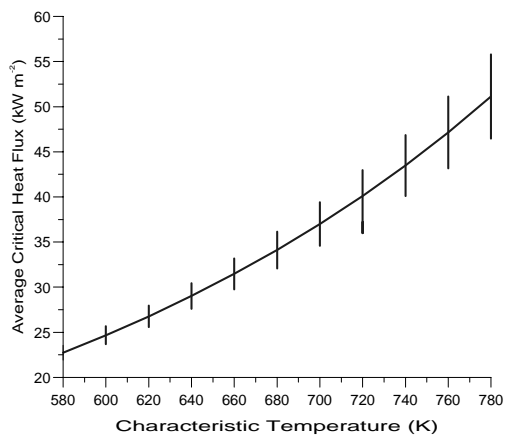

(b) Variation of average critical heat flux $\left(\overline{\mathcal{L}_{c r}}\right)$ with characteristic temperature. $Q_{s}=$ $2000\left(\mathrm{~kJ} \mathrm{~kg}^{-1}\right)$.

Figure 2. The calculation of average critical heat flux for non-charring materials $(c=0)$.

energy materials. Figure 3 (b) shows that the maximum efficiency for a given char fraction occurs for the lowest activation energy which is therefore an upper bound on the efficiency.

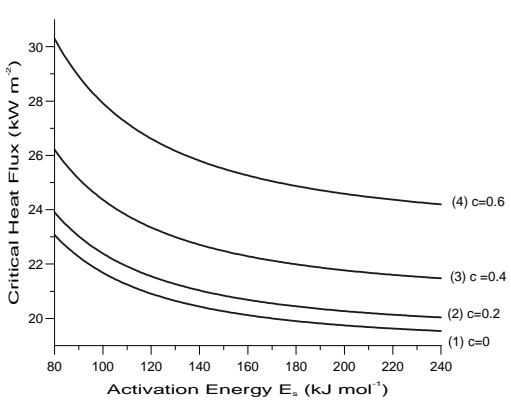

(a) Variation of critical heat flux $\left(\mathcal{L}_{c r}\right)$ with activation energy $\left(E_{s}\right)$ and char fraction $(c)$.

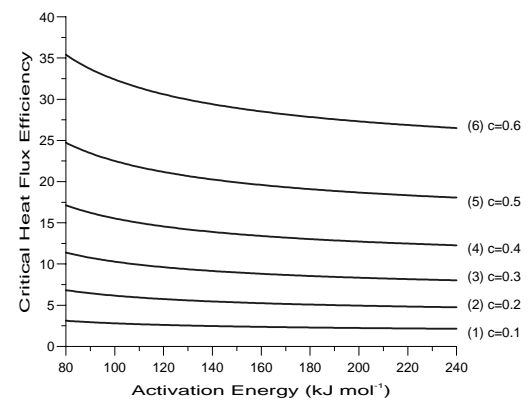

(b) Variation of critical heat flux efficiency $\left(\mathcal{E}_{\mathcal{L}_{c r}}\right)$ with activation energy $\left(E_{s}\right)$ and char fraction $(c)$.

Figure 3. The effect of char formation upon the critical heat flux. 


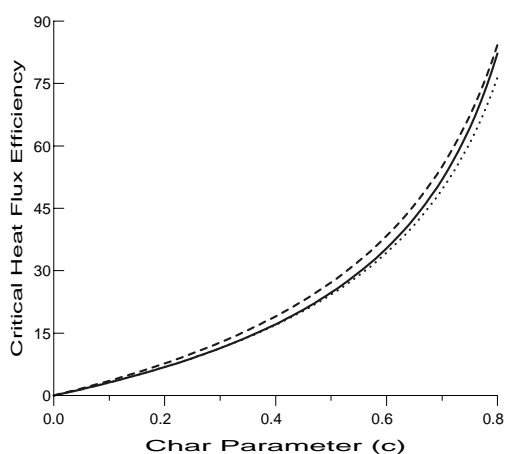

(a) Variation of critical heat flux efficiency $\left(\mathcal{E}_{\mathcal{L}_{c r}}\right)$ with char fraction $(c)$. Parameter values: $T_{c}$ : $580(\mathrm{~K})$, solid line, $680(\mathrm{~K})$, dotted line, $780(\mathrm{~K})$, dashed line.

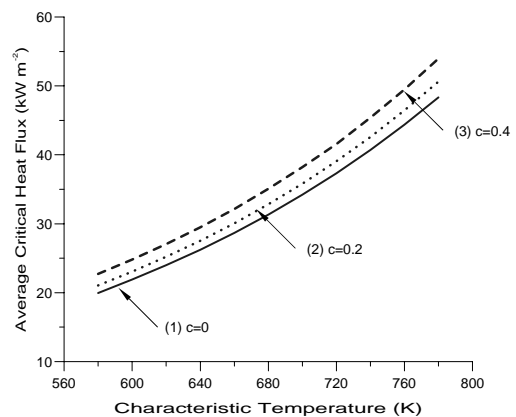

(b) Variation in average critical heat flux $\left(\overline{\mathcal{L}_{c r}}\right)$ with characteristic temperature $\left(T_{c}\right)$ and fraction of char formed $(c)$.

Figure 4. Characterizations of the efficiency of char formation in increasing the critical heat flux.

Figure 4 (a) shows how the efficiency depends upon the characteristic temperature. In view of the trends exhibited in figure 3 we take an activation energy $E_{s}=80\left(\mathrm{kJmol}^{-1}\right)$. The curves in this figure are therefore upper bounds on the behaviour for higher activation energies. When the char fraction is 0.4 the efficiency is modest, no more than $15 \%$. To achieve an efficiency of $50 \%$ a char fraction of approximately 0.70 is required. A material producing $100 \%$ char does not have a critical heat-flux. Therefore as the char-fraction increases towards unity the efficiency approaches a vertical asymptote. Thus there is a value of the char fraction at which it equals the fractional efficiency. These value are: $c=0.794\left(T_{c}=580 \mathrm{~K}\right)$, $c=0.812\left(T_{c}=680 \mathrm{~K}\right)$ and $c=0.785\left(T_{c}=780 \mathrm{~K}\right)$. Thus a considerable char fraction is required to significantly increase the critical heat flux.

For high density materials the efficiency of forming a certain fraction of char depends only weakly upon the characteristic temperature of the reaction. Calculations shows that the dependence is more noticeable for low density materials.

Figure 5 shows how the percentage char required to increase the average critical heat flux by $10 \%$, a very modest increase from a practical perspective, depends upon the density of the polymer and the endothermicity of the 
degradation reaction. The critical fraction decreases as the endothermicity decreases. However, even in the best case a char fraction of approximately 0.2 is required. There is only a very slight variation in the required char fraction with the polymer density.

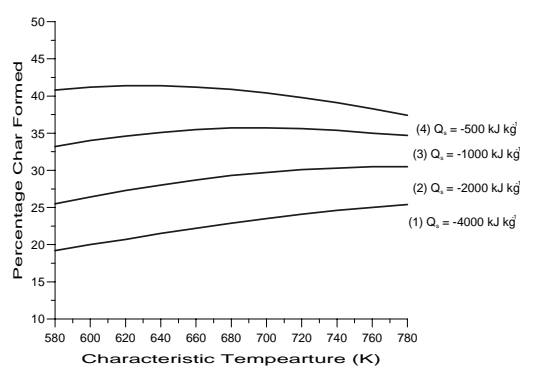

(a) Parameter values: $\rho=$ $500 \mathrm{~kg} \mathrm{~m}^{-3}$.

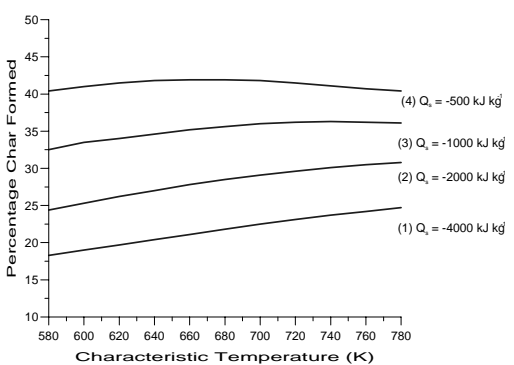

(b) Parameter values: $\rho=$ $2000 \mathrm{~kg} \mathrm{~m}^{-3}$.

Figure 5. Percentage char required to increase the average critical heat flux $\left(\overline{\mathcal{L}_{c r}}\right)$ by $10 \%$ over that of the comparison materials. Parameter values: activation energy, $E_{s}=80\left(\mathrm{kJmol}^{-1}\right)$.

In view of the trends displayed in figures 3-5 we conclude that modest amounts of char formation have negligible practical effect on the critical heat flux.

\subsubsection{Analytic results in the high activation energy limit}

The surface temperature at criticality $\left(T_{c r}^{*}\right)$ is obtained from equation (18) by setting the mass flux to its critical value $\left(\dot{m}^{*}=1\right)$. The critical heat flux is then obtained from equation (22) using the critical surface temperature. This gives

$$
\begin{aligned}
T_{c r}^{*}= & \frac{E_{s}^{*} T_{c}^{*}}{T_{c}^{*} \ln \left[\left(\frac{1-c}{m_{t}^{*}}\right) \cdot \frac{Y_{s}^{*} E_{s}^{*}}{T_{c}^{* 2}}\right]+E_{s}^{*}}, \\
\alpha_{s}\left(1-\alpha_{f}\right) \mathcal{L}_{c r}^{*}= & \left(T_{c r}^{*}-1\right)+\epsilon_{s} \sigma^{*} T_{c r}^{*^{4}}+\frac{\left|Q^{*}\right| m_{t}^{*}}{1-c} \\
& -\alpha_{s} \sigma^{*}\left[1-\mathcal{F}_{f . s}+\mathcal{F}_{f . s} \epsilon_{f}\right] .
\end{aligned}
$$


In the high activation energy limit $\left(E_{s}^{*} \rightarrow \infty\right)$ these reduce to

$$
\begin{aligned}
T_{c r}^{*}= & T_{c}^{*}, \\
\alpha_{s}\left(1-\alpha_{f}\right) \mathcal{L}_{c r}^{*}= & \left(T_{c}^{*}-1\right)+\epsilon_{s} \sigma^{*} T_{c}^{*^{4}}+\frac{\left|Q^{*}\right| m_{t}^{*}}{1-c} \\
& -\alpha_{s} \sigma^{*}\left[1-\mathcal{F}_{f . s}+\mathcal{F}_{f . s} \epsilon_{f}\right] .
\end{aligned}
$$

This limit is equivalent to replacing finite-rate chemistry by a thermal pyrolysis model in which the thermal pyrolysis temperature is identified with the characteristic temperature. The dimensional counterparts of equations (24$27)$ with $c=0$ have previously been obtained in the thermally-thin theory for thermoplastics[18].

The critical heat flux efficiency is given by

$$
\begin{aligned}
\mathcal{E}_{\mathcal{L}_{c r}^{*}}= & 100\left(\frac{\mathcal{L}_{c r}^{*}(c)}{\mathcal{L}_{c r}^{*}(c=0)}-1\right), \\
= & 100\left(\frac{\left|Q^{*}\right| m_{t}^{*}}{\left(T_{c}^{*}-1\right)+\epsilon_{s} \sigma^{*} T_{c}^{* 4}+\left|Q^{*}\right| m_{t}^{*}-\alpha_{s} \sigma^{*}\left(1-\mathcal{F}_{f . s}+\mathcal{F}_{f . s} \epsilon_{f}\right)}\right) \\
& \cdot \frac{c}{1-c} \\
= & 100\left(\frac{\left|Q^{*}\right| m_{t}^{*}}{\alpha_{s}\left(1-\alpha_{f}\right) \mathcal{L}_{c r}^{*}(c=0)}\right) \cdot \frac{c}{1-c} .
\end{aligned}
$$

Equation (30) is linear in the char fraction when $0<c \ll 1$. Since $\sigma^{*}=0.05$ and $T_{c}^{*}>0.939$, the term between the parenthesis in equation (29) is smaller than 1. Thus for small $c$ the fractional efficiency is smaller than the char fraction. For sufficiently large $c$ the fractional efficiency is greater than the char fraction. Thus there is a critical value of $c$ at which these are equal. This is the transition point after which the efficiency increases more rapidly than the char fraction.

\subsection{Maximum mass loss rate (peak heat release rate)}

Figure 6 shows the maximum mass loss rate (peak heat release rate) efficiency for two char-forming scenarios. As they are qualitatively the same we describe figure 6 (a). Three regions may be distinguished: $20 \leq$ $\mathcal{L}\left(\mathrm{kWm}^{-2}\right) \leq 22$, in which the efficiency is $-60 ; 22.5 \leq \mathcal{L}\left(\mathrm{kWm}^{-2}\right) \leq 30$, in which the efficiency changes from -100 to -96 ; and, $30.5 \leq \mathcal{L}\left(\mathrm{kWm}^{-2}\right)$, when the efficiency is -60 . The striking feature is that in the first and third regions the maximum mass loss rate efficiency is $-100 \cdot c$.

This behaviour can be understood by identifying the physical significance of the transition points between the regions. The first region is terminated 


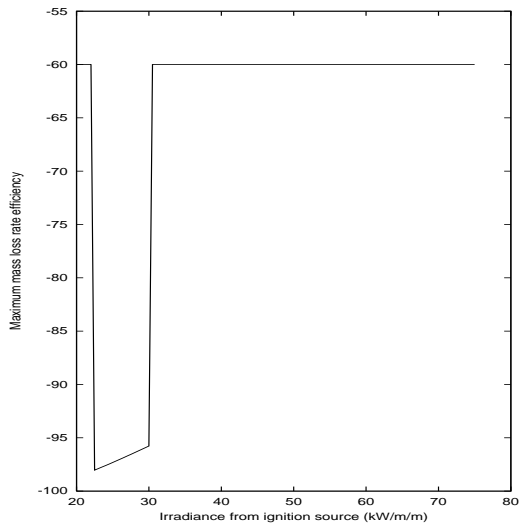

(a) Parameter values: char fraction, $c=0.6$.

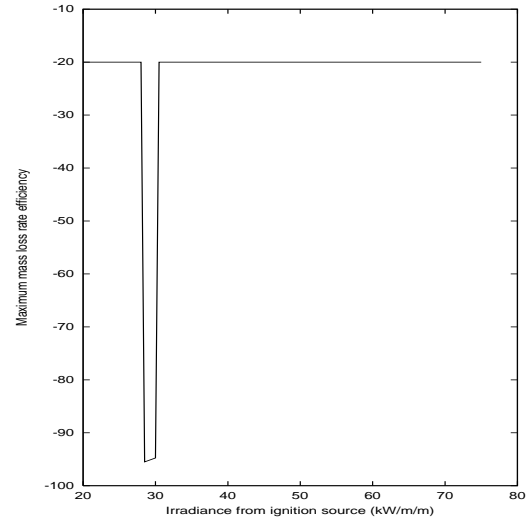

(b) Parameter values: char fraction, $c=0.2$

Figure 6. Variation of maximum mass loss rate efficiency with the external irradiance $(\mathcal{L})$.

when the irradiance equals the critical heat flux for the comparison material. The second region terminates at the critical heat flux for the charforming material. Therefore in the first region both the char-forming and comparison materials are heated by subcritical irradiance. Similarly both are heated by supercritical irradiances in the third region. In the second region the irradiances are subcritical for the char-forming material but are supercritical for the comparison material.

Therefore in the first and third regions the value for $T_{f}^{*}$ is the same for both materials. As char fraction does not appear in the steady-state energy equation, equation (22), it follows that in these regions the steady-state temperature $\left(T^{*}\right)$ is the same for both types of material. Hence

$$
\begin{aligned}
\mathcal{E}_{\dot{m}_{\text {max }}^{*}}\left(\mathcal{L}^{*}\right) & =100\left(\frac{\dot{m}_{\text {max }}^{*}\left(\mathcal{L}^{*}, c\right)}{\dot{m}_{\text {max }}^{*}\left(\mathcal{L}^{*}, 0\right)}-1\right) \\
& =100\left(\frac{\frac{1-c}{m_{t}^{*}} \cdot \frac{Y_{s}^{*} E_{s}^{*}}{T_{c}^{* 2}} \exp \left[E_{s}^{*}\left(\frac{1}{T_{c}^{*}}-\frac{1}{T^{*}\left(\mathcal{L}^{*}\right)}\right)\right]}{\frac{1}{m_{t}^{*}} \cdot \frac{Y_{s}^{*} E_{s}^{*}}{T_{c}^{* 2}} \exp \left[E_{s}^{*}\left(\frac{1}{T_{c}^{*}}-\frac{1}{T^{*}\left(\mathcal{L}^{*}\right)}\right)\right]}-1\right) \\
& =-100 \cdot c .
\end{aligned}
$$

In the second region the char-forming material is being heated subcritically and therefore the steady mass-loss rate is less than unity. The compari- 
son material is being heated supercritically and consequently has a much higher steady mass loss rate. Thus, at least under marginally supercritical conditions,

$$
\frac{\dot{m}_{\max }^{*}\left(\mathcal{L}^{*}, c\right)}{\dot{m}_{\max }^{*}\left(\mathcal{L}^{*}, 0\right)} \approx 0
$$

and the efficiency is bounded below by -100 . The efficiency becomes less negative as the irradiance is increased as the char-forming material approaches supercriticality.

\section{Transient Results}

In this section we consider the transient formulation of our model, equations (15-21). Figure 7 shows the transient evolution of the system for the comparison material and two char-forming materials.

Figure 7 (a) shows temperature-time curves. The comparison material ignites first, char-formation impedes ignition. The pre-ignition curves for the cases $c=0$ and $c=0.2$ 'peel off' that for $c=0.5$. Figure 7 (b) shows that in this region there is little pyrolysis, and therefore $\mathcal{C}_{1}^{*} \approx 0$ and $\mathcal{M}_{1}^{*} \approx 1$. Thus the energy equation, equation (17), does not depend upon char fraction in the pre-ignition region.

There are three post-ignition regions in the temperature-time curve [19], [20]. In the first the temperature increases rapidly until it reaches its equilibrium value in the corresponding steady-state theory. Fuel pyrolysis is negligible in this region.

In the second region the temperature continues to raise, increasing to its maximum value at the end of this region. Fuel consumption is no longer negligible and through most of this region the mass flux is decreasing. The end of the region corresponds to the extinction condition $\left(\dot{m}^{*}=1\right)$.

In the third region the flame is 'switched off'. The temperature decreases to its final value which is the equilibrium solution of equation (17) in the limit $\mathcal{M}_{1}^{*} \rightarrow 0$. Although the comparison material reaches the equilibrium value instantaneously in the char-forming systems the temperature decays towards the equilibrium value.

Figure 7 (c) shows the mass-loss rate-time curves. In these the peak value decreases linearly in proportion to the char fraction. The peak occurs a little after the transition point between the first and second post-ignition regions, in the region where pyrolysis has started to reduce the sample mass. Thus the maximum transient mass-loss rate is lower than the predicated steady-state value [19], [20]. 


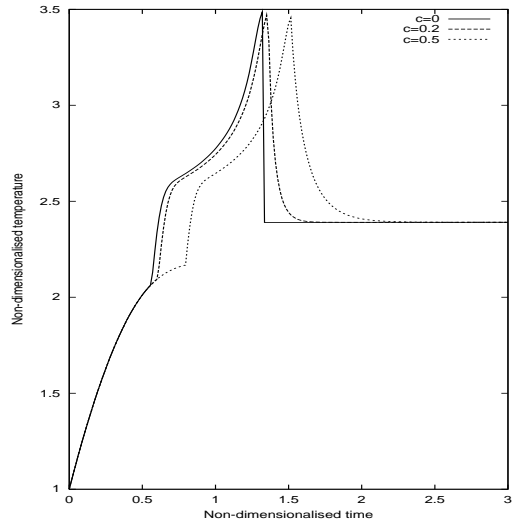

(a) Dimensionless temperature $\left(T_{s}\right)$

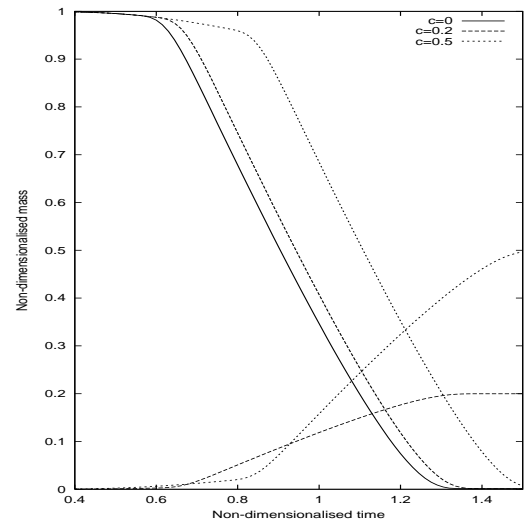

(b) Dimensionless polymer $\left(\mathcal{M}_{1}^{*}\right)$ and char $\left(\mathcal{C}_{1}^{*}\right)$ mass.

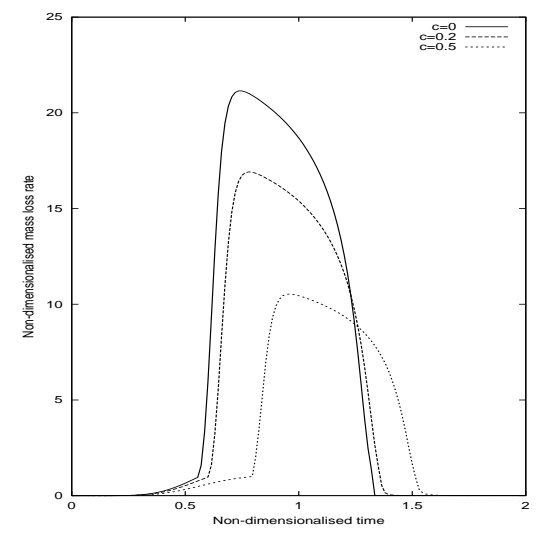

(c) Dimensionless mass-loss rate $\left(\dot{m}^{*}\right)$.

Figure 7. Transition evolution of the system. Parameter value: heat flux, $\mathcal{L}=30 \mathrm{kJm}^{-2}$.

Figure 8 shows how the efficiency depends upon the char fraction for low (a) and high (b) activation energies. The percentage efficiencies for the peak heat release rate, average heat release rate, and total heat released are $-100 \cdot c$. Of these, the effect upon the total heat release is the greatest. 


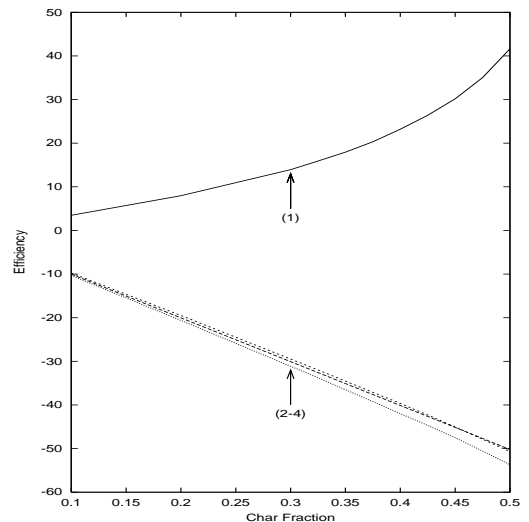

(a) Parameter values: activation energy, $E_{s}=80 \times 10^{3} \mathrm{Jmol}^{-1}$; heat flux, $\mathcal{L}=30 \times 10^{3} \mathrm{Jm}^{-2}$.

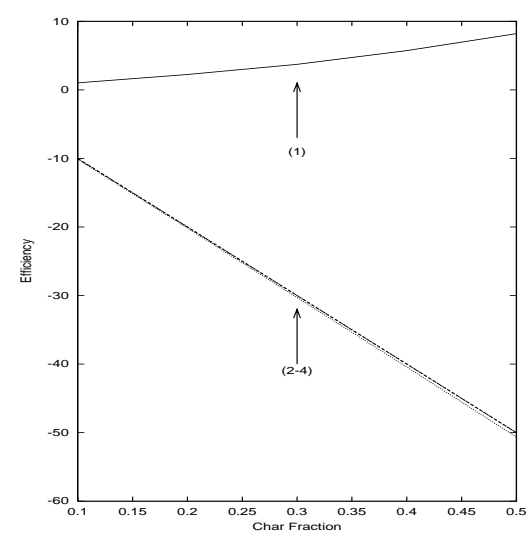

(b) Parameter values: activation energy, $E_{s}=200 \times 10^{3} \mathrm{Jmol}^{-1}$; heat flux, $\mathcal{L}=30 \times 10^{3} \mathrm{Jm}^{-2}$.

Figure 8. Variation of efficiency with char fraction: (1) time-to-ignition; (2) peak heat release rate; (3) average heat release rate; (4) total heat released.

For small values of the char fraction the time-to-ignition efficiency varies linearly with $c$. For a fixed value of the char fraction the efficiency is highest for the low activation energy material (a), see also figure (9). Charformation increases the ignition time because at a given temperature $\left(T_{s}\right)$ the mass-flux from the surface is reduced by a factor $(1-c)$. Thus a higher temperature must be reached before ignition occurs and consequently the ignition time is higher than that of the comparison material.

As the activation energy increases the temperature range over which pyrolysis occurs decreases and the rate of change of pyrolysis increases more rapidly in the vicinity of the characteristic temperature. Eventually this change becomes exponentially large, swamping the reduction due to char formation. Thus time-to-ignition efficiency reduces as the activation energy increases and in the thermal pyrolysis limit $\left(E_{s} \rightarrow \infty\right)$ the time-to-ignition for the comparison material and any char-forming material are equal. Figure 9 (b) shows that the time-to-ignition efficiency decreases with increasing heat flux. The peak heat release rate, average heat release rate and total heat release efficiency show negligible variation with the heat flux. (A figure is not used to illustrate this). 


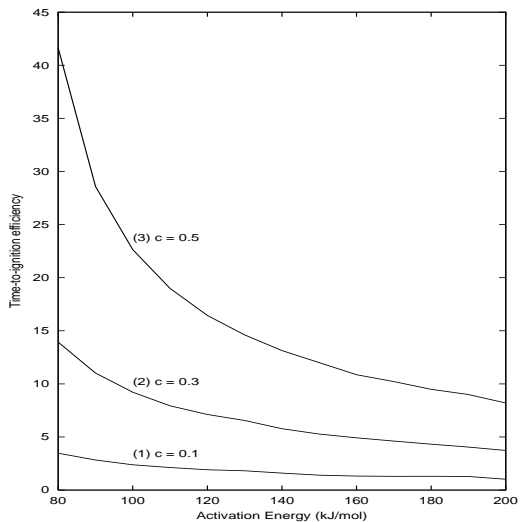

(a) Parameter value: heat flux, $\mathcal{L}=30 \times 10^{3} \mathrm{Jm}^{-2}$.

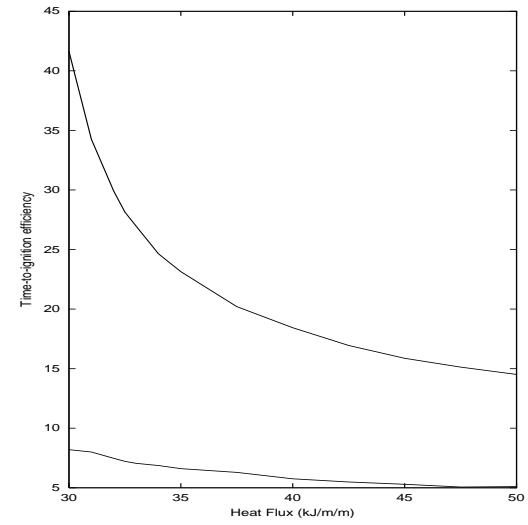

(b) Parameter values: activation energy, $E_{s}=80 \mathrm{kJmol}^{-1}$ (upper curve) and $E_{s}=200 \mathrm{kJmol}^{-1}$ (lower curve).

Figure 9. The dependence of the time-to-ignition efficiency upon activation energy (a) and heat flux (b).

\section{Discussion}

The total heat release rate efficiency is given by

$$
\begin{aligned}
\mathcal{E}_{\mathcal{H R}^{T} \mathcal{R}_{\mathrm{T}}} & =100\left(\frac{\mathcal{M}_{1, i g n}(c)-\mathcal{M}_{1, \text { ext }}(c)}{\mathcal{M}_{1, \text { ign }}(c=0)-\mathcal{M}_{1, \text { ext }}(c=0)}-1\right), \\
& \approx 100\left(\frac{\mathcal{M}_{1, i g n}(c)-c \cdot \mathcal{M}_{1, \text { ign }}(c)}{\mathcal{M}_{1, i g n}(c=0)}-1\right), \\
& =100\left(\frac{\mathcal{M}_{1, i g n}(c)-\mathcal{M}_{1, i g n}(c=0)}{\mathcal{M}_{1, i g n}(c=0)}-c\right),
\end{aligned}
$$

in which it is assumed that the mass at extinction in the comparison material is zero. A good approximation to the mass at ignition is to equate it to the initial mass which gives $\mathcal{E}_{\mathcal{H} \mathcal{R} \mathcal{R}_{\mathrm{T}}} \approx-100 \cdot c$. In fact a small amount of mass is pyrolysed prior to ignition. This pre-ignition mass loss increases with char fraction, because ignition occurs at a higher temperature with increasing char fraction. Thus $\mathcal{M}_{1, i g n}(c)<\mathcal{M}_{1, i g n}(c=0)$ and the total heat release rate efficiency should be slightly better than $-100 \cdot c$; the devi- 
ation increasing with increasing char fraction. This behaviour can be seen in figure 8 .

The average heat release rate efficiency is given by

$$
\begin{aligned}
\mathcal{E}_{\mathcal{H} \mathcal{R} \mathcal{R}_{\mathrm{A}}} & =100\left(\frac{\mathcal{M}_{1, i g n}(c)-\mathcal{M}_{1, \text { ext }}(c)}{\mathcal{M}_{1, i g n}(0)-\mathcal{M}_{1, \text { ext }}(0)} \cdot \frac{t_{1, i g n}(0)-t_{1, \text { ext }}(0)}{t_{1, i g n}(c)-t_{1, \text { ext }}(c)}-1\right) \\
& =100\left(\frac{\mathcal{E}_{\mathcal{H} \mathcal{R} \mathcal{R}_{\mathrm{T}}}-\mathcal{E}_{t_{b}}}{100+\mathcal{E}_{t_{b}}}\right)
\end{aligned}
$$

where $\mathcal{E}_{t_{b}}$ is the burn-time efficiency. The burn time for a char-forming material is slightly lower than that for the comparison material and thus the burn-time efficiency is negative. The burn-time efficiency decreases with increasing char fraction. For the parameter values used in figure 8 (a) the change is from -0.6 (when $c=0.1$ ) to -6 (when $c=0.5$ ). The corresponding values in figure 8 (b) are -0.01 and -1.14 . Thus in equation (39) the burn-time efficiency is small, when it is zero the average heat release rate efficiency and the total heat release efficiency are equal.

It follows from equation (39) that if $100+\mathcal{E}_{t_{b}}>0$ then $\mathcal{E}_{\mathcal{H} \mathcal{R} \mathcal{R}_{\mathrm{A}}}>\mathcal{E}_{\mathcal{H}} \mathcal{R R}_{\mathrm{T}}$. Thus char formation is more effective at decreasing the total heat release rather than the average rate of heat release. However, since the burn time efficiency is small the difference is negligible in practical terms.

It is not possible to derive an expression for the transient peak heat release rate efficiency. However a good approximation is given by the steady-state theory.

\section{Conclusions}

A model has been developed for the combustion of thermally-thin charring materials that form thermally stable char non-competitively. The efficiency of a charring material is defined with respect to a non-charring material with the same physical and chemical properties. Steady-state and transient models have been investigated, the former is a useful starting point as some analysis of the model is possible. In neither formulation does the char density have any effect upon the evolution of the system: the major mechanism by which char formation reduces flammability is through the reduction of volatiles entering the gas-phase.

The effect of char formation upon the critical heat flux has been investigated using the steady-state model. For small values of the char fraction the fractional efficiency is lower than the char fraction. There is a critical value of the char fraction at which it is equal to the fractional efficiency, after which the fractional efficiency increases rapidly with char fraction. 
However, the critical value is very high. In the high activation energy limit $\left(E_{s} \rightarrow \infty\right)$ the critical heat flux efficiency is proportional to $c /(1-c)$. Noncompetitive char-formation is ineffective at increasing the critical heat flux unless $c$ is large. This is shown in figures 3-5.

A simple argument shows that in the steady-state model the maximum mass loss rate (peak heat release rate) efficiency is $-100 \cdot c$. Thus even modest amounts of char lead to significant reductions in the mass loss rate. In the transient model it has been shown that the average mass loss (average heat release rate) and the total heat released are approximately $-100 \cdot c$, with the reduction in the total heat released being slightly greater than in the other two measures. These results are independent of the imposed heat flux and are a consequence of char being formed non-competitivalye. In competitive schemes the efficiency is expected to vary with the heat flux.

We hope that future work on charring materials will be able to use our results as a basis for a systematic quantification of the contribution made by the various mechanisms noted in section 1 .

\section{Acknowledgments}

This work was carried out whilst MIN was supported by the EPSRC (GR/L28142).

The authors thank Professor A.R. Horrocks (Bolton Institute) for suggesting that critical mass flux models be used to investigate the flammability of char-forming materials.

\section{Appendix Nomenclature}

To save space parameters that were defined in our earlier papers have been obmitted. These can be found elsewhere[21]. The subscript $i$ is used to represent either $s$, a property of the solid phase, or $g$, a property of the gas phase. The subscript $c r$ pertains to criticality.

$\begin{array}{lll}\mathcal{C}_{1} & \text { The mass of char in the system. } & (\mathrm{kg})\end{array}$

$\mathcal{C}_{1}^{*} \quad$ The non-dimensionalized mass of char in the system. $\mathcal{C}^{*}=\frac{\mathcal{C}}{\rho_{s} V_{0}}$

$E_{s}^{*} \quad$ Non-dimensionalized activation energy. $E_{s}^{*}=\frac{E}{R T_{a}}$

$\mathcal{E}_{P} \quad$ The percentage efficiency of char formation for a given $\quad(-)$ flammability parameter $P$ Defined by equation (5).

$\mathcal{H R R}_{A} \quad$ Average heat release rate during the burn. Defined by equation (14). 
$\mathcal{H R}_{T}$ Total heat released during the burn.

$\mathcal{L}^{*} \quad$ Non-dimensionalized irradiance from the ignition source.

$$
\mathcal{L}^{*}=\frac{\mathcal{L}}{\chi T_{a}}
$$

$\mathcal{M}_{1, \text { ext }}$ The mass of polymer remaining at extinction.

$\mathcal{M}_{1, \text { ign }}$ The mass of polymer at ignition.

$P(c) \quad$ The value of a flammability parameter for a given char fraction.

$Q_{s}^{*} \quad$ Non-dimensionalized modulus of the heat of reaction.

$$
Q_{s}^{*}=\frac{Q_{s}}{c_{p_{s}} T_{a}}
$$

$T_{c}^{*} \quad$ Non-dimensionalized characteristic temperature.

$$
T_{c}^{*}=T_{c} / T_{a}
$$

$T_{f}^{*} \quad$ Non-dimensionalized flame temperature.

Defined by equation (20).

$T F^{*} \quad$ Non-dimensionalized temperature of the flame under superritical conditions. $T F^{*}=T F / T_{a}$

$Y_{s}^{*} \quad$ Non-dimensionalized pre-exponential factor for the polymer $(-)$ degradation reaction. $Y_{s}^{*}=\frac{\mathcal{H} c_{p_{s}} \rho_{s} V_{s}}{S \chi T_{a}}$

c Fraction of char formed by the degradation of a unit mass of polymer.

$c_{p}^{*} \quad$ Ratio of heat-capacity of the char to that of the polymer. $c_{p}^{*}=\frac{c_{p_{c}}}{c_{p_{s}}}$

$k \quad$ The rate constant for a first order chemical reaction.

$m_{t}^{*}$ A non-dimensionalized mass transfer number.

$$
m_{t}^{*}=\frac{c_{p_{s}}}{\chi} \cdot m_{c r}^{\prime \prime}
$$

$t_{b} \quad$ The burn-time. $t_{b}=t_{e x t}-t_{i g n}$.

$t_{\text {ext }} \quad$ The extinction time.

$t_{\text {ign }} \quad$ The ignition time.

$\sigma^{*} \quad$ Non-dimensionalized radiative heat transfer coefficient.

$$
\sigma^{*}=\sigma \cdot \frac{T_{a}^{3}}{\chi}
$$

Unless otherwise specified we use the following typical values:

$E=80\left(\mathrm{kJmol}^{-1}\right), \mathcal{F}_{\text {f.s }}=1, \mathcal{H}=1 / 60\left(\mathrm{Ks}^{-1}\right), \mathcal{L}=50 \times 10^{3}\left(\mathrm{Wm}^{-2}\right)$, $Q_{s}=10^{3}\left(\mathrm{kJkg}^{-1}\right), R=8.31441\left(\mathrm{JK}^{-1} \mathrm{~mol}^{-1}\right), S=0.0625\left(\mathrm{~m}^{2}\right), T_{a}=$ $298(\mathrm{~K}), T_{c}=580(\mathrm{~K}), T F=1500(\mathrm{~K}), c=0, c_{p_{s}}=1 \times 10^{3}\left(\mathrm{JK}^{-1} \mathrm{~kg}^{-1}\right)$, $\dot{m}_{c r}^{\prime \prime}=2.5 \times 10^{-3}\left(\mathrm{~kg} \mathrm{~m}^{-2} \mathrm{~s}^{-1}\right), \delta=0.001(\mathrm{~m}), \epsilon_{f}=0.1, \epsilon_{s}=\alpha_{s}=1, \rho_{s}=$ $2 \times 10^{3}\left(\mathrm{kgm}^{-3}\right), \sigma=5.67 \times 10^{-8}\left(\mathrm{Wm}^{-2} \mathrm{~K}^{-4}\right)$, and $\chi=30\left(\mathrm{Wm}^{-2} \mathrm{~K}^{-1}\right)$. 


\section{References}

1. V. Babrauskas and R.D. Peacock. Heat release rate: The single most important variable in fire hazard. Fire Safety Journal, 18:255-272, 1992.

2. C.H. Bamford, J. Crank, and D.H. Malan. On the combustion of wood. Part I. Proceedings of the Cambridge Phil. Soc., 42:166-182, 1946.

3. C. Di Blasi. Modelling and simulation of combustion processes of charring and non-charring solid fuels. Progress in Energy and Combustion Science, 19:71-104, 1993.

4. M. Le Bras, G. Camino, S. Bourbigot, and R. Delobel, editors. Fire Retardancy of Polymers: The use of Intumescene. The Royal Society of Chemistry, Cambridge, 1998.

5. G. Camino, L. Cota, E. Casorati, G. Bertelli, and R. Locatelli. The oxygen index method in fire retardance studies of polymeric materials. Journal of Applied Polymer Science, 35:1863-1876, 1988.

6. Y. Chen, M.A. Delichatsios, and V. Motevalli. Material pyrolysis properties, part I: An integral model for one-dimensional transient pyrolysis of charring and noncharring materials. Combustion Science and Technology, 88:309-328, 1993.

7. Y. Chen, V. Motevalli, and M.A. Delichatsios. Material pyrolysis properties, part I: Methodology for the derivation of pyrolysis properties for charring materials. Combustion Science and Technology, 104:401-425, 1995.

8. D. Drysdale. An Introduction to Fire Dynamics. John Wiley and Sons, 1987.

9. T. Hatakeyama and F.X. Quinn. Thermal Analysis: Fundamentals and Applications to Polymer Science. John Wiley \& Sons Ltd, 1994.

10. A.R. Horrocks. Developments in flame retardants for heat and fire resistant textiles - the role of char formation and intumescence. Polymer Degradation and Stability, 54:143-154, 1996.

11. C.E. Anderson Jr, D.E. Ketchum, and W.P. Mountain. Thermal conductivity of intumescent chars. Journal of Fire Sciences, 6:390-410, November/December 1988.

12. B. Kandola, A.R. Horrocks, D. Price, and G.C. Coleman. Flame retardant treatments of cellulose and their mechanism of cellulose pyrolysis. Journal of Macromolecular Science - Reviews in Macormolecular Chemistry and Physics, C36:721$794,1996$.

13. T. Kashiwagi. Polymer combustion and flammability - role of the condensed phase. In Twenty-Fifth Symposium (International) On Combustion, pages 1423-1437, 1994.

14. H-C Kung. A mathematical model of wood pyrolysis. Combustion and Flame, 18:185-195, 1972.

15. C.H. Leung, J.E.J. Staggs, J. Brindley, and A.C. McIntosh. Modelling of a one dimensional ablation process with char formation. Combustion Science and Technology, 119:301-329, 1996.

16. C.H. Leung, J.E.J. Staggs, J. Brindley, and A.C. McIntosh. Modelling of polymer ablation including a substrate base. Combustion Science and Technology, 126:53$70,1997$.

17. T. Matsumtot, T. Fujiwara, and J. Kondo. Nonsteady thermal decomposition of plastics. In Twelfth Symposium (International) on Combustion, pages 515-524, 1969.

18. M.I. Nelson, J. Brindley, and A.C. McIntosh. The dependence of critical heat flux on fuel and additive properties: A critical mass flux model. Fire Safety Journal, 24:107-130, 1995 . 
19. M.I. Nelson, J. Brindley, and A.C. McIntosh. Ignition properties of thermally thin materials in the cone calorimeter: A critical mass flux model. Combustion Science and Technology, 113-114:221-241, 1996.

20. M.I. Nelson, J. Brindley, and A.C. McIntosh. Ignition properties of thermally thin thermoplastics - The effectiveness of inert additives in reducing flammability. Polymer Degradation and Stability, 54:255-267, 1996.

21. M.I. Nelson, J. Brindley, and A.C. McIntosh. The effect of heat sink additives on the ignition and heat release properties of thermally thin thermoplastics. Fire Safety Journal, 28:67-94, 1997.

22. S.J. Ritchie, K.D. Steckler, A. Hamins, T.G. Cleary, J.C. Yang, and T. Kashiwagi. The effect of sample size on the heat release rate of charring materials. In Fire Safety Science: Proceedings of the Fifth International Symposium, pages 177-188, 1997.

23. M. Sibulkin. Heat of gasification for pyrolysis of charring materials. In Fire Safety Science: Proceedings of the First International Symposium, pages 391-400. Hemisphere, 1997.

24. J.E.J. Staggs. Modelling pyrolysis of char forming polymers. In Interflam' 99, volume 1, pages 167-179, Greenwich, London, 1999. Interscience Communications Limited.

25. E.D. Weil, W. Zhu, N. Patel, and S.M. Mukhopadhyay. A systems approach to flame retardancy and comments on modes of action. Polymer Degradation and Stability, 54:125-136, 1996.

26. R.H. Whiteley. Some comments on the measurement of ignitability and on the calculation of 'critical heat flux'. Fire Safety Journal, 21:177-183, 1993.

27. C.A. Wilkie, M. Suzuki, X. Dong, C. Deacon, J.A. Chandrasiri, and T.J. Xue. Grafting to achieve flame retardancy. Polymer Degradation and Stability, 54:117$124,1996$.

28. R. Yuen, R. Casey, G. de Vahl Davis, E. Leonardi, G.H. Yeoh, V. Chandrasekaran, and S.J. Grubits. A three-dimensional mathematical model for the pyrolysis of wet wood. In Fire Safety Science: Proceedings of the Fifth International Symposium, pages 189-200, 1997. 


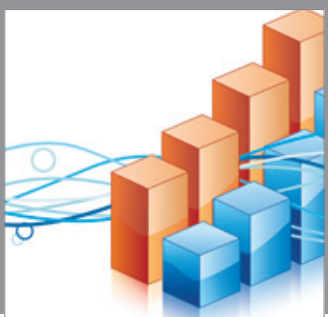

Advances in

Operations Research

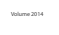

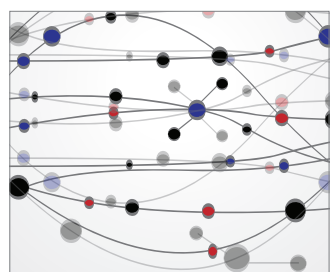

\section{The Scientific} World Journal
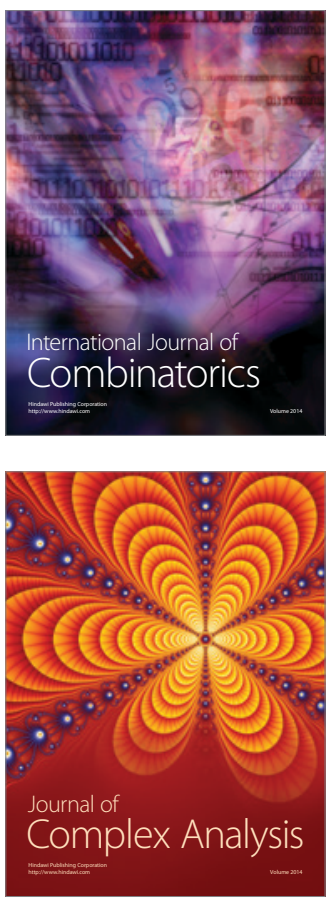

International Journal of

Mathematics and

Mathematical

Sciences
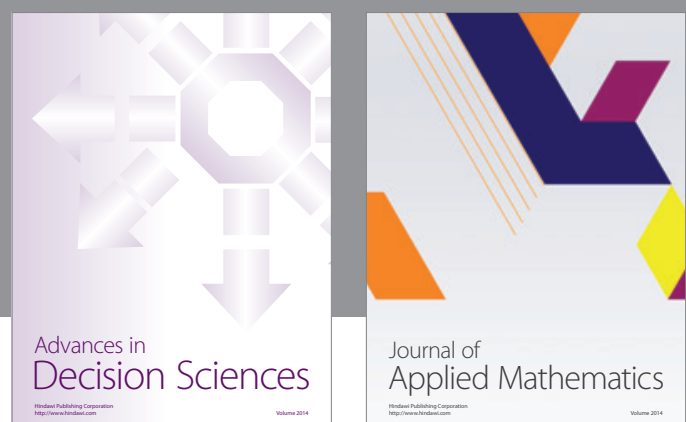

Journal of

Applied Mathematics
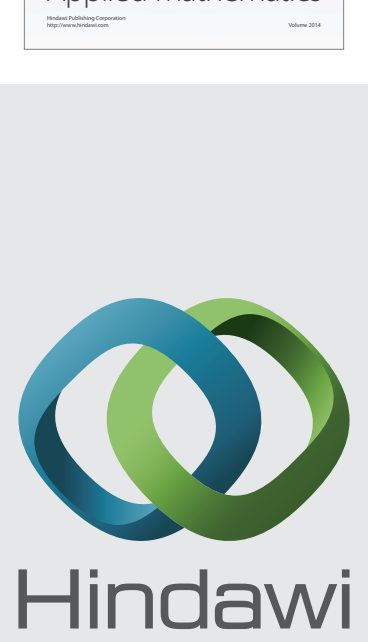

Submit your manuscripts at http://www.hindawi.com
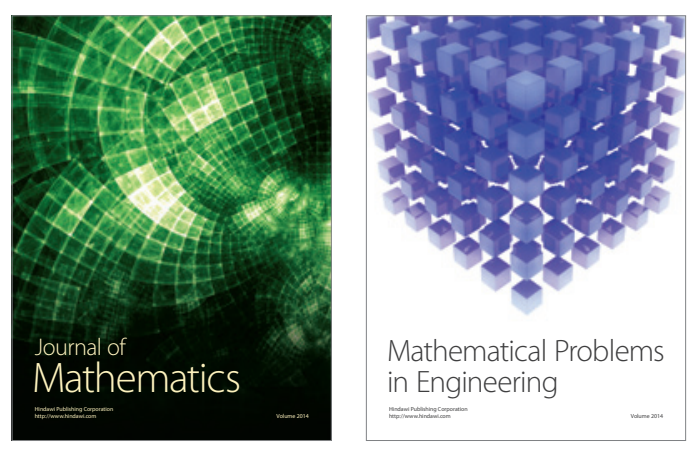

Mathematical Problems in Engineering
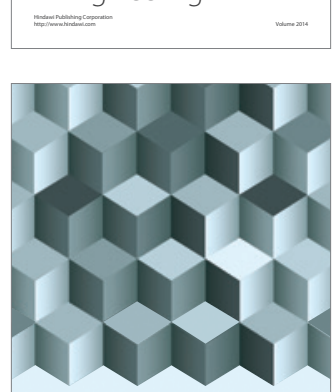

Journal of

Function Spaces
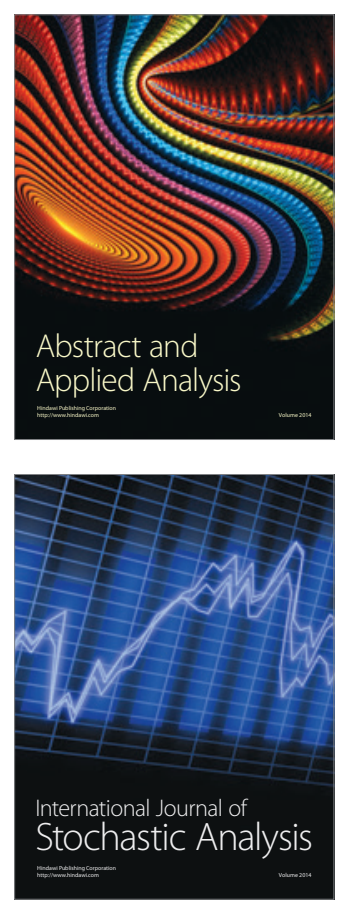

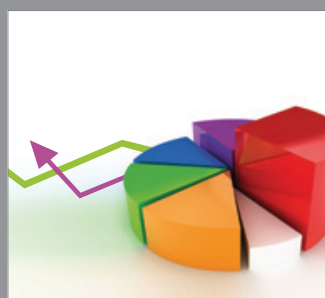

ournal of

Probability and Statistics

Promensencen
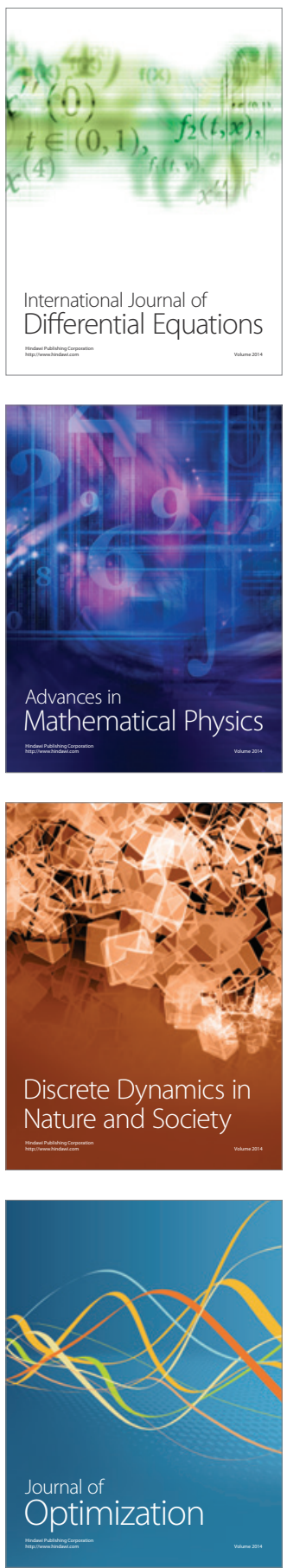